\title{
Existence of positive periodic solutions for Liénard equations with an indefinite singularity of attractive type
}

Shiping $\mathrm{Lu}^{1 *}$ and Xingchen $\mathrm{Yu}{ }^{1}$

\section{"Correspondence: \\ lushiping88@sohu.com \\ ${ }^{1}$ College of Math and Statistics, \\ Nanjing University of Information \\ Science and Technology, Nanjing, \\ China}

\begin{abstract}
In this paper, we study the periodic problem for the Liénard equation with an indefinite singularity of attractive type$$
x^{\prime \prime}+f(x) x^{\prime}+\varphi(t) x+\frac{r(t)}{x^{\mu}}=0
$$

where $f:(0,+\infty) \rightarrow R$ is continuous and may have singularities at zero, $r, \varphi: R \rightarrow R$ are $T$-periodic functions, and $\mu$ is a positive constant. Using the method of upper and lower functions, we obtain some new results on the existence of positive periodic solutions to the equation.
\end{abstract}

Keywords: Boundary value problem; Periodic solution; Singularity; Upper and lower functions

\section{Introduction}

As is well known, differential equations with singularities have a wide range of applications in physics, mechanics, and biology [1-6]. In the past years, many mathematical researchers focused their attention on the equations with singularities [7-20]. As is widely acknowledged, the paper [18] by Lazer and Solimini is a major milestone for the study of periodic problem to second-order differential equations with singularities. In that paper, the existence of periodic solutions was investigated for the singular equations

$$
x^{\prime \prime}(t)+\frac{1}{x^{\alpha}(t)}=h(t)
$$

(the singularity of attractive type) and

$$
x^{\prime \prime}(t)-\frac{1}{x^{\alpha}(t)}=h(t)
$$

(the singularity of repulsive type), where $h: R \rightarrow R$ is a continuous periodic function. Using topological degree methods, together with the method of lower and upper functions, they obtained that a necessary and sufficient condition for the existence of positive periodic

(c) The Author(s) 2018. This article is distributed under the terms of the Creative Commons Attribution 4.0 International License (http://creativecommons.org/licenses/by/4.0/), which permits unrestricted use, distribution, and reproduction in any medium, provided you give appropriate credit to the original author(s) and the source, provide a link to the Creative Commons license, and indicate if changes were made. 
solutions to (1.1) is $\int_{0}^{T} h(s) d s>0$. Furthermore, assuming that $\alpha \geq 1$, a necessary and sufficient condition for the existence of positive periodic solutions to (1.2) is $\int_{0}^{T} h(s) d s<0$. For $\alpha \in(0,1)$ (weak singularity condition), some equations like (1.2) were given in [18], where $h(t)$ have negative mean values, but the equations have no $T$-periodic solution. After that, many papers focused on the periodic problem for some second-order differential equations with singularities of repulsive type [21-24]. Among them, the singular term was allowed to have a weak singularity (i.e., $\alpha \in(0,1))$. Compared with the singularity of repulsive type, the attractive case did not attract much attention of mathematical researchers. Even so, there are still quite a few papers that focus on the study of periodic solutions for the equations with attractive singularities $[7,16,17,20]$. For example, Mawhin [7] considered the problem of periodic solutions to the Liénard equation with a attractive singularity suggested by the fundamental example

$$
\left(\left|x^{\prime}\right|^{p-2} x^{\prime}\right)^{\prime}+f(x) x^{\prime}+\frac{l}{x^{\mu}}=h(t),
$$

where $p>1, l>0$, and $\mu>0$ are constants, and $h \in L^{\infty}(0, T)$. Using the method of upper and lower functions, they obtained that $\int_{0}^{T} h(s) d s>0$ is a necessary and sufficient condition for the existence of positive periodic solutions for equation (1.3). Hakl and Torres [16] obtained sufficient conditions guaranteeing the existence of positive solutions to the periodic problem associated to the equation of Rayleigh-Plesset type

$$
\begin{aligned}
& x^{\prime \prime}+f(x(t)) x^{\prime}(t)+\varphi(t) x^{\sigma}+\frac{g_{1}}{x^{\nu}}-\frac{g_{2}}{x^{\gamma}}=0, \\
& x(0)=x(T), \quad x^{\prime}(0)=x^{\prime}(T),
\end{aligned}
$$

where $g_{1}, g_{2}, \sigma$ are nonnegative constants, $c, \mu, v, \gamma$ are real numbers, and $h_{0} \in L([0, T] ; R)$. For the case of $\delta=1, g_{1}>0$, and $g_{2}=0$, they obtained the following result.

Theorem 1.1 Let $\delta=1, g_{1}>0$, and $g_{2}=0$. If $\bar{\varphi}<0$ and

$$
\frac{T}{4} \int_{0}^{T}[\varphi(s)]_{+} d s \int_{0}^{T}[\varphi(s)]_{-} d s<\int_{0}^{T}[\varphi(s)]_{-} d s-\int_{0}^{T}[\varphi(s)]_{+} d s,
$$

then there exists at least one positive solution to problem (1.4)-(1.5).

It is easy to see that in either equation (1.3) or the equation in problem (1.4)-(1.5), the singular terms are all autonomous. Despite the fact that there are many papers focusing on the equation with nonautonomous singularity term of repulsive type [21-25], equations with nonautonomous singularity term of attractive type seem to receive little attention. We have found only the paper by Hakl and Zamora [17], who studied the following equations with a singularity of attractive type:

$$
x^{\prime \prime}+\frac{g(t)}{x^{\lambda}}=h(t) x^{\delta}
$$

where $\lambda>0, \delta \in[0,1), g, h \in L([0, T] ; R)$, and $g$ is a nonnegative function. Using a continuation theorem of coincidence degree theory, they obtained a new result on the existence of positive periodic solutions to (1.6). However, the exponent $\delta$ in the power function $x^{\delta}$ 
is required to satisfy $\delta \in[0,1)$, and there is no friction term of Liénard type $f(x) x^{\prime}$ in (1.6). For other recent developments and applications in this field, we refer the reader to [16, 23, 26-31]. Inspired by the papers mentioned, the aim of this paper is to study the periodic problem

$$
\begin{aligned}
& x^{\prime \prime}+f(x) x^{\prime}+\varphi(t) x+\frac{r(t)}{x^{\mu}}=0, \\
& x(0)=x(T), \quad x^{\prime}(0)=x^{\prime}(T),
\end{aligned}
$$

where $f$ belongs to $C((0, \infty) ; R)$ and may have singularities at zero, $r, \varphi: R \rightarrow R$ are $T$ periodic functions with $r, \varphi \in L([0, T] ; R)$, and $\mu$ is a positive constant. Since $r(t)$ is a timevarying function, (1.7) describes the nature processes more accurately. Observe that in the case where $r(t)$ is equal to zero for some subinterval of $[0, T]$, the singularity can disappear. So the singularity $\frac{r(t)}{x^{\mu}}$ in equation (1.7) is said to be of indefinite attractive type. Using the method of lower and upper functions, we obtain some new results on the existence of positive solutions to boundary value problem (1.7)-(1.8). The significance is that the methods for constructing lower and upper functions in [16] cannot be directly applied to (1.7), since the singularity term $\frac{r(t)}{x^{\mu}}$ in (1.7) is nonautonomous, and $r(t)$ may be equal to zero at some $t \in[0, T]$.

Remark 1.1 In this paper, a function $u:[0, T] \rightarrow(0,+\infty)$ is said to be a positive solution to problem (1.7)-(1.8) if $u:[0, T] \rightarrow R_{+}$is absolutely continuous together with its first derivative on $[0, T]$ and satisfies (1.8) together with (1.7) almost everywhere on $[0, T]$. From this definition, $r, \varphi: R \rightarrow R$ being $T$-periodic, we can easily find that if $u_{0}:[0, T] \rightarrow R$ is a positive solution to boundary value problem (1.7)-(1.8), then $\tilde{u}: R \rightarrow R$, which is a $T$-periodic extension of $u_{0}(t)$, is a positive $T$-periodic solution to (1.7). Thus, the existence of positive periodic solutions to (1.7) is equivalent to the existence of positive solutions to boundary value problem (1.7)-(1.8).

For convenience, in the end of this introduction, we give some notations used throughout the paper:

$R_{+}=(0,+\infty), R_{+}^{0}=[0,+\infty),[x]_{+}=\max \{x, 0\},[x]_{-}=\max \{-x, 0\} ; A C^{1}([0, T] ; R)$ is the set of functions $u:[0, T] \rightarrow R$ such that $u$ and $u^{\prime}$ are absolutely continuous; $\bar{p}=\frac{1}{T} \int_{0}^{T}|p(s)| d s$ for $p \in L([0, T] ; R)$.

\section{Preliminary lemmas}

The method of lower and upper functions is one of the most widely used methods in nonlinear analysis. Its main idea goes back at least to Picard. Many mathematical researchers have obtained rich results by using this method. For a complete historical review of the method, we refer to the monograph [32]. Now, we give the definitions of upper and lower functions.

Definition 2.1 A function $\alpha \in A C^{1}([0, T] ; R)$ is called a lower function to problem (1.7)(1.8) if $\alpha(t)>0$ for every $t \in[0, T]$ and

$$
\begin{aligned}
& \alpha^{\prime \prime}(t)+f(\alpha(t)) \alpha^{\prime}(t)+\varphi(t) \alpha(t)+\frac{r(t)}{\alpha^{\mu}(t)} \geq 0, \quad \text { a.e. } t \in[0, T], \\
& \alpha(0)=\alpha(T), \quad \alpha^{\prime}(0) \geq \alpha^{\prime}(T) .
\end{aligned}
$$


Definition 2.2 A function $\beta \in A C^{1}([0, T] ; R)$ is called an upper function to problem (1.7)(1.8) if $\beta(t)>0$ for every $t \in[0, T]$ and

$$
\begin{aligned}
& \beta^{\prime \prime}(t)+f(\beta(t)) \beta^{\prime}(t)+\varphi(t) \beta(t)+\frac{r(t)}{\beta^{\mu}(t)} \leq 0, \quad \text { a.e. } t \in[0, T], \\
& \beta(0)=\beta(T), \quad \beta^{\prime}(0) \leq \beta^{\prime}(T) .
\end{aligned}
$$

The following proposition can be found in [32] (or a more general case in [33]).

Proposition 2.1 Let $\alpha$ and $\beta$ be lower and upper functions to problem (1.7)-(1.8) such that

$$
\alpha(t) \leq \beta(t), \quad t \in[0, T] .
$$

Then there exists a positive solution $u$ to problem (1.7)-(1.8) such that

$$
\alpha(t) \leq u(t) \leq \beta(t), \quad t \in[0, T] .
$$

We further show some auxiliary results obtained by Hakl, Torres and Zamora [16].

Given $x_{1} \in R_{+}$and $x_{0} \in R_{+}^{0}$ as fixed constants and the operator $K: C^{1}([0, T] ; R) \rightarrow$ $C^{1}([0, T] ; R)$ defined by

$$
K(u)(t)=x_{1}+x_{0}(u(t)-\min \{u(s): s \in[0, T]\}), \quad t \in[0, T],
$$

we consider the auxiliary problem

$$
\begin{aligned}
& x^{\prime \prime}(t)+f(K(x)(t)) x^{\prime}(t)=q(t), \quad \text { a.e. } t \in[0, T], \\
& x(0)=0, \quad x(T)=0,
\end{aligned}
$$

where $f \in C\left(R_{+} ; R\right)$ and $q \in L([0, T] ; R)$. By a solution to problem (2.2)-(2.3) we understand a function $u \in A C^{1}([0, T] ; R)$ that satisfies (2.2) almost everywhere on $[0, T]$ and (2.3).

Lemma 2.1 ([16]) For every solution u to problem

$$
\begin{aligned}
& x^{\prime \prime}(t)+\lambda f(K(x)(t)) x^{\prime}(t)=\lambda q(t), \quad \text { a.e. } t \in[0, T], \\
& x(0)=0, \quad x(T)=0,
\end{aligned}
$$

with $\lambda \in(0,1]$, we have the estimate

$$
M-m \leq \frac{T}{4} \max \left\{\int_{0}^{T}[q(s)]_{+} d s, \int_{0}^{T}[q(s)]_{-} d s\right\},
$$

where $M=\max \{u(t): t \in[0, T]\}, m=\min \{u(t): t \in[0, T]\}$.

Lemma 2.2 ([16]) For all $x_{1} \in R_{+}, x_{0} \in R_{+}^{0}$, and $q \in L([0, T] ; R)$, there exists a solution $u(t)$ to problem (2.2)-(2.3). Furthermore,

$$
u^{\prime}(T)-u^{\prime}(0)=\int_{0}^{T} q(s) d s
$$

and (2.6) is fulfilled. 
Lemma 2.3 If $h, \alpha \in L([0, T], R)$ and $\alpha(t) \geq 0$ for a.e. $t \in[0, T]$, then

$$
\lim _{n \rightarrow+\infty} \int_{0}^{T}\left[h(s)-\frac{\alpha(s)}{n}\right]_{+} d s=\int_{0}^{T}[h(s)]_{+} d s .
$$

Proof Let

$$
h_{n}(t)= \begin{cases}\frac{\alpha(t)}{n}, & h(t)>\frac{\alpha(t)}{n} \\ h(t), & h(t) \leq \frac{\alpha(t)}{n}\end{cases}
$$

Then we have

$$
h(t)=h_{n}(t)+\left[h(t)-\frac{\alpha(t)}{n}\right]_{+} .
$$

Moreover,

$$
\int_{0}^{T} h(s) d s=\int_{0}^{T} h_{n}(s) d s+\int_{0}^{T}\left[h(s)-\frac{\alpha(s)}{n}\right]_{+} d s
$$

By the definition of $h_{n}(t)$ we obtain

$$
\lim _{n \rightarrow+\infty} h_{n}(t) \rightarrow f(t), \quad \text { a.e. } t \in[0, T],
$$

where

$$
\begin{gathered}
f(t)= \begin{cases}h(t), & \text { a.e. } t \in E_{-}(h), \\
0, & \text { a.e. } t \in E_{+}(h),\end{cases} \\
E_{-}(h)=\{t \in[0, T]: h(t) \leq 0\}, E_{+}(h)=\{t \in[0, T]: h(t)>0\}, \text { and } \\
-[h(t)]_{-} \leq h_{n}(t) \leq h(t) .
\end{gathered}
$$

By the Lebesgue dominated convergence theorem we get

$$
\lim _{n \rightarrow+\infty} \int_{0}^{T} h_{n}(t) d t=\int_{0}^{T} f(t) d t=-\int_{0}^{T}[h(t)]_{-} d t .
$$

Substituting this into (2.8), we obtain

$$
\lim _{n \rightarrow+\infty} \int_{0}^{T}\left[h(s)-\frac{\alpha(s)}{n}\right]_{+} d s=\int_{0}^{T}[h(s)]_{+} d s .
$$

The proof is complete.

\section{Main results}

\subsection{Construction of lower function}

In this section, we use the notations

$$
Y_{+}=\int_{0}^{T}[y(s)]_{+} d s,
$$




$$
Y_{-}=\int_{0}^{T}[y(s)]_{-} d s
$$

where $y \in L([0, T] ; R)$.

Theorem 3.1 Let $\varphi, r \in L([0, T], R)$ be such that $\operatorname{essinf}\{r(t): t \in[0, T]\}>0$. Then there exists a lower function $\alpha$ to problem (1.7)-(1.8) satisfying $0<\alpha(t)<1$.

Proof Let $r_{1}>0$ be such that

$$
\operatorname{essinf}\{r(t): t \in[0, T]\} \geq r_{1} \text {. }
$$

Consider the periodic problem

$$
x^{\prime \prime}+f(x) x^{\prime}+\varphi(t) x+\frac{r_{1}}{x^{\mu}}=0, \quad x(0)=x(T), \quad x^{\prime}(0)=x^{\prime}(T) .
$$

By Corollary 2.13 in [16] (with $h_{0}(t)=-\varphi(t), \rho_{0}(x)=x, g(x)=\frac{r_{1}}{x^{\mu}}, c$ sufficiently large, and $\left.x_{0} \in(0,1)\right)$ there exists a lower function $\alpha$ to periodic problem (3.1) such that $0<\alpha(t) \leq x_{0}$ for $t \in[0, T]$. Obviously, the same $\alpha$ is also a lower function to (1.7)-(1.8).

Theorem 3.2 Let $\varphi, r \in L([0, T], R), r(t) \geq 0$ a.e. $t \in[0, T]$ and $\bar{r}>0$. Suppose $\frac{T}{4} \times$ $\int_{0}^{T}[\varphi(s)]_{-}(s) d s<1$. Then there exists a lower function $\alpha$ to problem (1.7)-(1.8) such that $0<\alpha(t)<1$ for $t \in[0, T]$.

Proof Let

$$
y(t, x)=-\varphi(t)-\frac{r(t)}{x^{\mu+1}} \quad \text { for }(t, x) \in[0, T] \times(0,+\infty) .
$$

Then we have

$$
Y(x)=\int_{0}^{T} y(s, x) d s=-T \bar{\varphi}-\frac{T \bar{r}}{x^{\mu+1}} .
$$

Obviously, for a constant $c$ satisfying $c>-\bar{\varphi}$, there must exist a constant $a_{1} \in(0,1)$ such that, for any $x \in\left(0, a_{1}\right)$, we have

$$
Y(x)<0 .
$$

Since $Y(x)=Y_{+}(x)-Y_{-}(x)$, we arrive at $Y_{+}(x)<Y_{-}(x)$ for $x \in\left(0, a_{1}\right)$. By a direct calculation, for $x \in\left(0, a_{1}\right)$, we get

$$
Y_{+}(x) \leq \int_{0}^{T}[\varphi(s)]_{-} d s
$$

Since $Y(x)=Y_{+}(x)-Y_{-}(x)$, we obtain the following inequality as $x \rightarrow 0^{+}$:

$$
Y_{-}(x)=Y_{+}(x)-Y(x) \geq-Y(x)=T \bar{\varphi}+\frac{T \bar{r}}{x^{\mu+1}} \rightarrow+\infty .
$$


So, for a sufficiently small constant $a_{1} \in(0,1)$, we have the following equality for $x \in\left(0, a_{1}\right)$ :

$$
1+\frac{T}{4} Y_{-}(x)>\frac{1}{1-\frac{T}{4} \int_{0}^{T}[\varphi(s)]_{-} d s} .
$$

Choose a constant $x_{2} \in\left(0, a_{1}\right)$, and let $x_{1}=\left(1-\frac{T}{4} Y_{+}\left(x_{2}\right)\right) x_{2}$. Since $\frac{T}{4} \int_{0}^{T}[\varphi(s)]_{-} d s<1$ by assumption, we have $1-\frac{T}{4} Y_{+}\left(x_{2}\right)>0$, which ensures that $x_{1}$ make sense. As the first case, we suppose $Y_{+}\left(x_{2}\right)>0$.

Put

$$
\begin{aligned}
& x_{0}=\frac{4\left(x_{2}-x_{1}\right)}{T Y_{-}\left(x_{2}\right) Y_{+}\left(x_{2}\right)}, \\
& q(t)=Y_{-}\left(x_{2}\right)\left[y\left(t, x_{2}\right)\right]_{+}-Y_{+}\left(x_{2}\right)\left[y\left(t, x_{2}\right)\right]_{-}, \quad t \in[0, T] .
\end{aligned}
$$

Now we obtain

$$
\int_{0}^{T} q(s) d s=0
$$

By Lemma 2.2 there exists a solution $u$ to (2.2)-(2.3) such that (2.6) and (2.7) hold.

Using (2.6) and (2.7), we get

$$
\begin{aligned}
& M-m \leq \frac{T}{4} Y_{+}\left(x_{2}\right) Y_{-}\left(x_{2}\right), \\
& u^{\prime}(0)=u^{\prime}(T)
\end{aligned}
$$

where the constants $M$ and $m$ are defined in Lemma 2.1.

Put $\alpha(t)=K(u)(t)=x_{1}+x_{0}(u(t)-\min \{u(s): s \in[0, T]\})$. Then equation (2.2) can be written as

$$
\alpha^{\prime \prime}(t)+f(\alpha(t)) \alpha^{\prime}(t)=x_{0} Y_{-}\left(x_{2}\right)\left[y\left(t, x_{2}\right)\right]_{+}-x_{0} Y_{+}\left(x_{2}\right)\left[y\left(t, x_{2}\right)\right]_{-}
$$

for almost every $t \in[0, T]$. Besides, according to (3.3), (3.5), and the definition of $\alpha(t)$, we arrive at

$$
x_{1} \leq \alpha(t) \leq x_{2}, \quad t \in[0, T]
$$

Then, using (3.6), we get

$$
\alpha(0)=\alpha(T), \quad \alpha^{\prime}(0)=\alpha^{\prime}(T) .
$$

In addition, by the definition of $x_{1}, x_{2}$ and by (3.2) we obtain

$$
\begin{aligned}
x_{2}-x_{1} & =x_{2}-x_{2}\left(1-\frac{T}{4} Y_{+}\left(x_{2}\right)\right) \\
& \leq x_{2}-x_{2}\left(1-\frac{T}{4} \int_{0}^{T}[\varphi(s)]_{-} d s\right)
\end{aligned}
$$




$$
\begin{aligned}
& \leq x_{2}-\frac{x_{2}}{1+\frac{T}{4} Y_{-}\left(x_{2}\right)}=\frac{\frac{T}{4} Y_{-}\left(x_{2}\right) x_{2}}{1+\frac{T}{4} Y_{-}\left(x_{2}\right)} \\
& \leq \frac{T}{4} Y_{-}\left(x_{2}\right) x_{2}\left(1-\frac{T}{4} Y_{+}\left(x_{2}\right)\right) \\
& =\frac{x_{1}}{1-\frac{T}{4} Y_{+}\left(x_{2}\right)}\left(1-\frac{T}{4} Y_{+}\left(x_{2}\right)\right) \frac{T}{4} Y_{-}\left(x_{2}\right) \\
& =\frac{T}{4} Y_{-}\left(x_{2}\right) x_{1} .
\end{aligned}
$$

From the definition of $x_{1}, x_{2}$, we arrive at

$$
x_{2}-x_{1}=\frac{T}{4} x_{2} Y_{+}\left(x_{2}\right)
$$

Using (3.3) and (3.10), we get

$$
x_{0} Y_{+}\left(x_{2}\right)=\frac{4\left(x_{2}-x_{1}\right)}{T Y_{-}\left(x_{2}\right)} \leq x_{1}
$$

Relations of (3.3) and (3.11) imply

$$
x_{0} Y_{-}\left(x_{2}\right)=\frac{4\left(x_{2}-x_{1}\right)}{T Y_{+}\left(x_{2}\right)}=x_{2}
$$

By (3.8), (3.12), (3.13) we obtain

$$
x_{0} Y_{+}\left(x_{2}\right) \leq \alpha(t) \leq x_{0} Y_{-}\left(x_{2}\right), \quad t \in[0, T]
$$

Substituting (3.14) into (3.7), we get

$$
\alpha^{\prime \prime}(t)+f(\alpha(t)) \alpha^{\prime}(t) \geq \alpha(t) y\left(t, x_{2}\right), \quad \text { a.e. } t \in[0, T]
$$

By (3.8) we have

$$
-\frac{r(t)}{\alpha^{\mu+1}(t)} \leq-\frac{r(t)}{x_{2}^{\mu+1}}
$$

Moreover,

$$
y\left(t, x_{2}\right)=-\varphi(t)-\frac{r(t)}{x_{2}^{\mu+1}} \geq-\varphi(t)-\frac{r(t)}{\alpha^{\mu+1}(t)}
$$

Substituting this into (3.15), we arrive at

$$
\alpha^{\prime \prime}(t)+f(\alpha(t)) \alpha^{\prime}(t)+\varphi(t) \alpha(t)+\frac{r(t)}{\alpha^{\mu}(t)} \geq 0, \quad \text { a.e. } t \in[0, T]
$$

Consequently, (3.9) and (3.17) ensure that $\alpha(t)$ is a lower function to problem (1.7)-(1.8) such that $0<\alpha(t)<1$ for $t \in[0, T]$. 
We further consider the case of $Y_{+}\left(x_{2}\right)=0$. For this case, it is easy to see that

$$
y\left(t, x_{2}\right) \leq 0
$$

which means

$$
-\varphi(t)-\frac{r(t)}{x_{2}^{\mu+1}} \leq 0
$$

that is,

$$
\varphi(t) x_{2}+\frac{r(t)}{x_{2}^{\mu}} \geq 0
$$

Obviously, $\alpha(t)=x_{2}$ is a lower function to problem (1.7)-(1.8), and $0<\alpha(t)<1$ for $t \in$ $[0, T]$.

\subsection{Construction of upper function}

Theorem 3.3 Let $r, \varphi \in L([0, T], R)$ be such that $\operatorname{ess} \sup \{r(t): t \in[0, T]\}<+\infty$. Let, moreover,

$$
\frac{T}{4} \int_{0}^{T}[\varphi(t)]_{-} d s \int_{0}^{T}[\varphi(t)]_{+} d s \leq \int_{0}^{T}[\varphi(t)]_{-} d s-\int_{0}^{T}[\varphi(t)]_{+} d s .
$$

Then there exists an upper function $\beta(t)$ to problem (1.7)-(1.8), and $\beta(t)>1$ for $t \in[0, T]$.

Proof Since ess $\sup \{r(t): t \in[0, T]\}<+\infty$, there exists a constant $c_{1} \in R$ such that

$$
\operatorname{ess} \sup \{r(t): t \in[0, T]\} \leq c_{1}
$$

which results in

$$
\lim _{x \rightarrow \infty} \frac{r(t)}{x^{\mu+1}}=0 \quad \text { uniformly for a.e. } t \in[0, T] .
$$

Thus there exist $1<a_{1}(\varepsilon) \leq a_{2}(\varepsilon)<+\infty$ such that

$$
\frac{r(t)}{x^{\mu+1}} \leq \varepsilon \quad \text { for every } x \in\left[a_{1}(\varepsilon), a_{2}(\varepsilon)\right] \text {, a.e. } t \in[0, T]
$$

Let $\psi(t)=-\varphi(t)-\varepsilon$. By Lemma 2.3 we have

$$
\lim _{\varepsilon \rightarrow 0} \int_{0}^{T}[-\varphi(t)-\varepsilon]_{+} d s=\int_{0}^{T}[\varphi(t)]_{-} d s
$$

namely,

$$
\lim _{\varepsilon \rightarrow 0} \int_{0}^{T}[\psi(t)]_{+} d s=\int_{0}^{T}[\varphi(t)]_{-} d s .
$$


We easily have

$$
\lim _{\varepsilon \rightarrow 0} \int_{0}^{T}[\psi(t)]_{-} d s=\int_{0}^{T}[\varphi(t)]_{+} d s
$$

Arguing as before, (3.18) is equal to

$$
\frac{T}{4} \int_{0}^{T}[\psi(t)]_{+} d s \int_{0}^{T}[\psi(t)]_{-} d s \leq \int_{0}^{T}[\psi(t)]_{+} d s-\int_{0}^{T}[\psi(t)]_{-} d s
$$

which implies $\bar{\psi} \geq 0$, that is, $\Psi_{+} \geq \Psi_{-} \geq 0$. As a first case, we suppose $\Psi_{-}>0$.

Put

$$
\begin{aligned}
& a_{0}=\frac{4\left(a_{2}-a_{1}\right)}{T \Psi_{+} \Psi_{-}}, \\
& q(t)=\Psi_{-}[\psi(t)]_{+}-\Psi_{+}[\psi(t)]_{-}, \quad \text { a.e. } t \in[0, T] .
\end{aligned}
$$

From the definition of $q(t)$ we have

$$
\int_{0}^{T} q(s) d s=0
$$

By Lemma 2.2 there exists a solution $u$ to problem (2.2)-(2.3) such that (2.6) and (2.7) hold. By (2.6) and (2.7) we get

$$
\begin{aligned}
& M-m \leq \frac{T}{4} \Psi_{+} \Psi_{-}, \\
& u^{\prime}(0)=u^{\prime}(T),
\end{aligned}
$$

where the constants $M$ and $m$ are defined in Lemma 2.1.

Let $\beta(t)=K(u)(t)=a_{1}+a_{0}(u(t)-\min \{u(s): s \in[0, T]\})$. The function $\beta$ satisfies

$$
\beta^{\prime \prime}(t)+f(\beta(t)) \beta^{\prime}(t)=a_{0} \Psi_{-}[\psi(t)]_{+}-a_{0} \Psi_{+}[\psi(t)]_{-}, \quad \text { a.e. } t \in[0, T] .
$$

Using (3.24), (3.25), and the definition of $\beta(t)$, we obtain

$$
\begin{aligned}
& a_{1} \leq \beta(t) \leq a_{2}, \\
& \beta(0)=\beta(T), \quad \beta^{\prime}(0)=\beta^{\prime}(T) .
\end{aligned}
$$

Putting $a_{2}=a_{1}+\frac{T}{4} a_{1} \Psi_{+}$and combining with (3.20), we arrive at

$$
a_{2} \Psi_{-} \leq a_{1} \Psi_{+}
$$

The definition of $a_{0}$ and (3.29) imply

$$
a_{2} \leq a_{0} \Psi_{+}, \quad a_{1}=a_{0} \Psi_{-} .
$$


From $a_{1} \leq \beta(t) \leq a_{2}$ we get

$$
a_{0} \Psi_{-} \leq \beta(t) \leq a_{0} \Psi_{+}
$$

Substituting (3.30) into (3.26), we arrive at

$$
\beta^{\prime \prime}(t)+f(\beta(t)) \beta^{\prime}(t) \leq \beta(t)[\psi(t)]_{+}-\beta(t)[\psi(t)]_{-}=\beta(t) \psi(t) \quad \text { a.e. } t \in[0, T],
$$

which, together with (3.19) and (3.27), gives

$$
\beta^{\prime \prime}(t)+f(\beta(t)) \beta^{\prime}(t) \leq \beta(t)\left(-\varphi(t)-\frac{r(t)}{\beta^{\mu+1}(t)}\right), \quad \text { a.e. } t \in[0, T],
$$

that is,

$$
\beta^{\prime \prime}(t)+f(\beta(t)) \beta^{\prime}(t)+\beta(t) \varphi(t)+\frac{r(t)}{\beta^{\mu}(t)} \leq 0, \quad \text { a.e. } t \in[0, T] .
$$

Consequently, by Definition 2.2, (3.33), and (3.28), we get that $\beta(t)$ is an upper function to problem (1.7) $-(1.8)$ and $\beta(t)>1$.

Now, we consider the case of $\Psi_{-}=0$. For this case, it is easy to see that $\psi(t) \geq 0$ for a.e. $t \in[0, T]$. By Lemma 2.2, when $q(t)=0$, there is a solution $u$ to (2.2)-(2.3) satisfying (3.24) and (3.25). Let $\beta(t)=K(u)(t)=a_{1}+a_{0}(u(t)-\min \{u(s): s \in[0, T]\})$. Then $\beta(t) \geq 0$ for all $t \in[0, T]$, and (3.26) can be rewritten as

$$
\beta^{\prime \prime}(t)+f(\beta(t)) \beta^{\prime}(t)=0 .
$$

Since $\beta(t) \psi(t) \geq 0$ for a.e. $t \in[0, T]$, we get

$$
\beta^{\prime \prime}(t)+f(\beta(t)) \beta^{\prime}(t) \leq \beta(t) \psi(t)=\beta(t)(-\varphi(t)-\varepsilon), \quad \text { a.e. } t \in[0, T],
$$

which, together with (3.19), yields

$$
\beta^{\prime \prime}(t)+f(\beta(t)) \beta^{\prime}(t) \leq \beta(t)\left(-\varphi(t)-\frac{r(t)}{\beta^{\mu+1}(t)}\right), \quad \text { a.e. } t \in[0, T],
$$

that is,

$$
\beta^{\prime \prime}(t)+f(\beta(t)) \beta^{\prime}(t)+\beta(t) \varphi(t)+\frac{r(t)}{\beta^{\mu}(t)} \leq 0, \quad \text { a.e. } t \in[0, T] .
$$

Consequently, by Definition 2.2, (3.28), and (3.34) we get that $\beta(t)$ is an upper function to problem (1.7)-(1.8) and $\beta(t)>1$.

Theorem 3.4 Let $\varphi, r \in L([0, T], R)$, and let $r(t) \geq 0$ for a.e. $t \in[0, T]$. Suppose that

$$
\frac{T}{4} \int_{0}^{T}[\varphi(s)]_{+} d s \int_{0}^{T}[\varphi(s)]_{-} d s<\int_{0}^{T}[\varphi(s)]_{-} d s-\int_{0}^{T}[\varphi(s)]_{+} d s .
$$

Then there exists an upper function $\beta(t)$ to problem (1.7)-(1.8), and $\beta(t)>1$. 
Proof Put $z(t, x)=-\varphi(t)-\frac{r(t)}{x^{\mu+1}},(t, x) \in[0, T] \times(0,+\infty)$. Then

$$
Z(x)=\int_{0}^{T} z(s, x) d s=-T \bar{\varphi}-\frac{T \bar{r}}{x^{\mu+1}}
$$

Using the definition of $Z(x)$, we easily obtain that $Z(x) \rightarrow-T \bar{\varphi}$ as $x \rightarrow+\infty$. Furthermore, by Lemma 2.3 we get

$$
Z_{+}(x) \rightarrow \Phi_{-}, \quad Z_{-}(x) \rightarrow \Phi_{+}, \quad x \rightarrow+\infty .
$$

The condition $r(t)>0$, a.e. $t \in[0, T]$, gives that

$$
Z_{+}(x) \leq \Phi_{-}, \quad Z_{-}(x) \geq \Phi_{+} \quad \text { for } x \in(0,+\infty) .
$$

Also, (3.36) implies that there exist a constant $\varepsilon_{0} \in(0,1)$ (small enough) and a constant $\rho \in(1,+\infty)$ (large enough) such that

$$
\Phi_{-}-\varepsilon_{0}<Z_{+}(x) \leq \Phi_{-}, \quad \Phi_{+} \leq Z_{-}(x)<\Phi_{+}+\varepsilon_{0}, \quad x \in(\rho,+\infty)
$$

and

$$
\frac{T}{4}\left(\int_{0}^{T}[\varphi(t)]_{+} d s+\varepsilon_{0}\right) \int_{0}^{T}[\varphi(t)]_{-} d s \leq \int_{0}^{T}[\varphi(t)]_{-} d s-\int_{0}^{T}[\varphi(t)]_{+} d s-2 \varepsilon_{0} .
$$

We consider the case $Z_{-}\left(a_{1}\right)>0$ as the first case. Put $a_{1} \in(\rho,+\infty)$ and $a_{2}=a_{1}+$ $\frac{T}{4} a_{1} Z_{+}\left(a_{1}\right)$. Then $a_{2} \in(\rho,+\infty)$. Let

$$
\begin{aligned}
& a_{0}=\frac{4\left(a_{2}-a_{1}\right)}{T Z_{+}\left(a_{1}\right) Z_{-}\left(a_{1}\right)}, \\
& q(t)=Z_{+}\left(a_{1}\right)\left[z\left(t, a_{1}\right)\right]_{-}-Z_{+}\left(a_{1}\right)\left[z\left(t, a_{1}\right)\right]_{-}, \quad \text { a.e. } t \in[0, T] .
\end{aligned}
$$

Clearly,

$$
\int_{0}^{T} q(s) d s=0
$$

By Lemma 2.2 there exists a solution $u$ to (2.2)-(2.3) satisfying (2.6) and (2.7). In view of (2.6) and (2.7), we get

$$
\begin{aligned}
& M-m \leq \frac{T}{4} Z_{+}\left(a_{1}\right) Z_{-}\left(a_{1}\right), \\
& u^{\prime}(0)=u^{\prime}(T),
\end{aligned}
$$

where the constants $M$ and $m$ are defined in Lemma 2.1. Put

$$
\beta(t)=K(u)(t)=a_{1}+a_{0}(u(t)-\min \{u(s): s \in[0, T]\}) .
$$


Then (2.2) can be rewritten as

$$
\beta^{\prime \prime}(t)+f(\beta(t)) \beta^{\prime}(t)=a_{0} Z_{-}\left(a_{1}\right)\left[z\left(t, a_{1}\right)\right]_{+}-a_{0} Z_{+}\left(a_{1}\right)\left[z\left(t, a_{1}\right)\right]_{-}
$$

a.e. $t \in[0, T]$. So

$$
\begin{aligned}
& 1<\rho<a_{1} \leq \beta(t) \leq a_{2}, \quad t \in[0, T] \\
& \beta(0)=\beta(T), \quad \beta^{\prime}(0)=\beta^{\prime}(T) .
\end{aligned}
$$

From (3.37), (3.38), and the definition of $a_{2}$ it follows that

$$
\begin{aligned}
a_{2} Z_{-}\left(a_{1}\right) & \leq a_{2}\left(\Phi_{+}+\varepsilon_{0}\right)=\left(a_{1}+\frac{T}{4} a_{1} Z_{+}\left(a_{1}\right)\right)\left(\Phi_{+}+\varepsilon_{0}\right) \\
& \leq a_{1}\left(1+\frac{T}{4} \Phi_{-}\right)\left(\Phi_{+}+\varepsilon_{0}\right) \\
& =a_{1}\left(\Phi_{+}+\varepsilon_{0}\right)+\frac{T}{4} a_{1} \Phi_{-}\left(\Phi_{+}+\varepsilon_{0}\right) \\
& <a_{1}\left(\Phi_{+}+\varepsilon_{0}\right)+\left(\Phi_{-}-\Phi_{+}-2 \varepsilon_{0}\right) a_{1} \\
& =a_{1}\left(\Phi_{-}-\varepsilon_{0}\right) \\
& <Z_{+}\left(a_{1}\right) a_{1} .
\end{aligned}
$$

Using (3.48) and the definition of $a_{0}$, we get

$$
a_{2} \leq a_{0} Z_{+}\left(a_{1}\right), \quad a_{1}=a_{0} Z_{-}\left(a_{1}\right)
$$

Because of $a_{1} \leq \beta(t) \leq a_{2}$, we have

$$
\beta(t) \leq a_{0} Z_{+}\left(a_{1}\right), \quad \beta(t) \geq a_{0} Z_{-}\left(a_{1}\right)
$$

Substituting this into (3.45), we obtain

$$
\beta^{\prime \prime}(t)+f(\beta(t)) \beta^{\prime}(t) \leq \beta(t)\left[z\left(t, a_{1}\right)\right]_{+}-\beta(t)\left[z\left(t, a_{1}\right)\right]_{-}=\beta(t) z\left(t, a_{1}\right)
$$

a.e. $t \in[0, T]$, that is,

$$
\beta^{\prime \prime}(t)+f(\beta(t)) \beta^{\prime}(t) \leq \beta(t)\left(-\varphi(t)-\frac{r(t)}{a_{1}^{\mu+1}}\right), \quad \text { a.e. } t \in[0, T]
$$

By the inequality $\beta(t) \geq a_{1}, t \in[0, T]$ (see (3.46)), we get that

$$
\beta^{\prime \prime}(t)+f(\beta(t)) \beta^{\prime}(t)+\beta(t) \varphi(t)+\frac{r(t)}{\beta^{\mu}(t)} \leq 0 \quad \text { a.e. } t \in[0, T]
$$

From (3.51) and (3.47) we see that $\beta(t)$ is an upper function to problem (1.7)-(1.8), and $\beta(t)>1$ for all $t \in[0, T]$. 
Now, we consider the remaining case $Z_{-}\left(a_{1}\right)=0$. Under this situation, we have $z\left(t, a_{1}\right) \geq$ 0 for $t \in[0, T]$, which implies

$$
-\varphi(t)-\frac{r(t)}{a_{1}^{\mu+1}} \geq 0
$$

that is,

$$
\varphi(t) a_{1}+\frac{r(t)}{a_{1}^{\mu}} \leq 0
$$

Obviously, $\beta(t)=a_{1}$ is an upper function to problem (1.7)-(1.8).

\subsection{Existence theorems}

Theorem 3.5 Let $\varphi, r \in L([0, T], R)$ be such that

$$
\operatorname{essinf}\{r(t): t \in[0, T]\}>0, \quad \operatorname{ess} \sup \{r(t): t \in[0, T]\}<+\infty
$$

Let, moreover,

$$
\frac{T}{4} \int_{0}^{T}[\varphi(t)]_{+} d s \int_{0}^{T}[\varphi(t)]_{-} d s \leq \int_{0}^{T}[\varphi(t)]_{-} d s-\int_{0}^{T}[\varphi(t)]_{+} d s .
$$

Then there exists at least one positive T-periodic solution to problem (1.7).

Proof By Theorems 3.1 and 3.3 there exist a lower function $0<\alpha(t)<1$ and an upper function $\beta(t)>1$. Therefore, the result can be obtained directly from Proposition 2.1 and Remark 1.1 in Sect. 1.

Example 3.1 Consider the following second-order differential equation:

$$
x^{\prime \prime}(t)+\frac{x^{\prime}(t)}{x^{3}}-(2+\sin t) x(t)+\frac{2+\sin t}{x^{\mu}(t)}=0
$$

where $\mu \in(0,+\infty)$ is a constant.

From the equation we see that $\varphi(t)=-2-\sin t$ and $r(t)=2+\sin t$; obviously, $r, \varphi \in$ $L([0, T], R)$, and $1 \leq r(t) \leq 3$. By direct calculation we get

$$
\begin{aligned}
& \int_{0}^{2 \pi}[\varphi(s)]_{+} d s=\int_{0}^{2 \pi} 0 d s=0 \\
& \int_{0}^{2 \pi}[\varphi(s)]_{-} d s=\int_{0}^{2 \pi}(2+\sin s) d s=4 \pi
\end{aligned}
$$

and we arrive at

$$
\begin{aligned}
& \frac{T}{4} \int_{0}^{T}[\varphi(t)]_{+} d s \int_{0}^{T}[\varphi(t)]_{-} d s=0 \\
& \int_{0}^{T}[\varphi(t)]_{-} d s-\int_{0}^{T}[\varphi(t)]_{+} d s=4 \pi
\end{aligned}
$$


Consequently, all the conditions of Theorem 3.5 are satisfied. So by this theorem we get that there exists at least one positive $T$-periodic solution to equation (3.52).

Theorem 3.6 Let $\varphi, r \in L([0, T], R)$ be such that

$$
r(t) \geq 0 \text {, a.e. } t \in[0, T]
$$

and

$$
\frac{T}{4} \int_{0}^{T}[\varphi(s)]_{+} d s \int_{0}^{T}[\varphi(s)]_{-} d s<\int_{0}^{T}[\varphi(s)]_{-} d s-\int_{0}^{T}[\varphi(s)]_{+} d s .
$$

Then there exists at least one positive T-periodic solution to problem (1.7).

Proof By Theorems 3.1 and 3.4 there exist a lower function $0<\alpha(t)<1$ and an upper function $\beta(t)>1$. Therefore, the result is a direct consequence of Proposition 2.1 and Remark 1.1 in Sect. 1.

Example 3.2 Consider the following second-order differential equation:

$$
x^{\prime \prime}(t)+\frac{x^{\prime}(t)}{x^{\frac{1}{3}}}-\left(1+\frac{1}{2} \sin t\right) x(t)+\frac{1+\frac{1}{2} \cos t}{x^{\mu}(t)}=0,
$$

where $\mu \in(0,+\infty)$ is a constant.

From the equation we see that $\varphi(t)=-1-\frac{1}{2} \sin t$ and $r(t)=1+\frac{1}{2} \cos t \geq 0$; obviously, $r, \varphi \in L([0, T], R)$. By direct calculation we have

$$
\begin{aligned}
& \int_{0}^{2 \pi}[\varphi(s)]_{+} d s=0 \\
& \int_{0}^{2 \pi}[\varphi(s)]_{-} d s=\int_{0}^{2 \pi}\left(1+\frac{1}{2} \sin s\right) d s=2 \pi
\end{aligned}
$$

and we arrive at

$$
\begin{aligned}
& \frac{T}{4} \int_{0}^{T}[\varphi(s)]_{+} d s \int_{0}^{T}[\varphi(s)]_{-} d s=0, \\
& \int_{0}^{T}[\varphi(s)]_{-} d s-\int_{0}^{T}[\varphi(s)]_{+} d s=2 \pi .
\end{aligned}
$$

Consequently, all the conditions of Theorem 3.6 are satisfied, and so by this theorem there exists at least one positive $T$-periodic solution to equation (3.53).

Theorem 3.7 Let $\varphi, r \in L([0, T], R)$. Suppose that the following assumptions hold:

(1) $r(t) \geq 0$ for a.e. $t \in[0, T], \bar{r}>0$, and $\operatorname{ess} \sup \{r(t)\}<+\infty$;

(2) $\frac{T}{4} \int_{0}^{T}[\varphi(s)]_{-} d s<1$;

(3) $\frac{T}{4} \int_{0}^{T}[\varphi(t)]_{+} d s \int_{0}^{T}[\varphi(t)]_{-} d s \leq \int_{0}^{T}[\varphi(t)]_{-} d s-\int_{0}^{T}[\varphi(t)]_{+} d s$.

Then there exists at least one positive T-periodic solution to problem (1.7). 
Proof By Theorems 3.2 and 3.3 there exist a lower function $0<\alpha(t)<1$ and an upper function $\beta(t)>1$. Therefore, the result is a direct consequence of Proposition 2.1 and Remark 1.1 in Sect. 1.

Example 3.3 Consider the following second-order differential equation:

$$
x^{\prime \prime}(t)-\frac{x^{\prime}(t)}{x^{\frac{1}{3}}}-(5-2 \sin t) x(t)+\frac{r(t)}{x^{\mu}(t)}=0,
$$

where $\mu \in(0,+\infty)$ is a constant, and $r: R \rightarrow R$ is $T$-periodic with

$$
r(t)= \begin{cases}-1, & t=-\pi, \\ 1+\sin t, & t \in(-\pi, \pi] .\end{cases}
$$

From equation (3.54) we see that $\varphi(t)=-5+2 \sin t$. Obviously, $\varphi, r \in L([0, T], R)$, and condition (1) is satisfied. By direct calculation we have

$$
\frac{2 \pi}{4} \int_{0}^{2 \pi}[-\varphi(s)]_{-} d s=\frac{2 \pi}{4} \int_{0}^{2 \pi} 0 d s=0<1
$$

This inequality implies that condition (2) is satisfied. Also, we can get the equalities

$$
\int_{0}^{2 \pi}[\varphi(s)]_{-} d s=\int_{0}^{2 \pi}(5-2 \sin s) d s=10 \pi
$$

and

$$
\int_{0}^{2 \pi}[\varphi(s)]_{+} d s=0
$$

which results in

$$
\begin{aligned}
& \frac{T}{4} \int_{0}^{T}[\varphi(t)]_{+} d s \int_{0}^{T}[\varphi(t)]_{-} d s=0 \\
& \int_{0}^{T}[\varphi(t)]_{-} d s-\int_{0}^{T}[\varphi(t)]_{+} d s=10 \pi .
\end{aligned}
$$

Consequently, all the conditions of Theorem 3.7 are satisfied, so by this theorem there exists at least one positive $T$-periodic solution to equation (3.54).

Theorem 3.8 Let $\varphi, r \in L([0, T], R)$ satisfy $\bar{r}>0$. Assume that the following conditions hold:

(1) $r(t) \geq 0$ for a.e. $t \in[0, T]$;

(2) $\frac{T}{4} \int_{0}^{T}[\varphi(s)]_{-}(s) d s<1$;

(3) $\frac{T}{4} \int_{0}^{T}[\varphi(s)]_{+} d s \int_{0}^{T}[\varphi(s)]_{-} d s<\int_{0}^{T}[\varphi(s)]_{-} d s-\int_{0}^{T}[\varphi(s)]_{+} d s$.

Then there exists at least one positive T-periodic solution to problem (1.7).

Proof By Theorems 3.2 and 3.4 there exist a lower function $0<\alpha(t)<1$ and an upper function $\beta(t)>1$. Therefore, the result follows immediately from Proposition 2.1 and Remark 1.1 in Sect. 1. 
Example 3.4 Consider the following second-order differential equation:

$$
x^{\prime \prime}(t)-f(x) x^{\prime}-\left(1+\frac{1}{2} \sin t\right) x(t)+\frac{r(t)}{x^{\mu}(t)}=0,
$$

where $f \in C((0,+\infty), R), \mu \in(0,+\infty)$ is a constant, and $r: R \rightarrow R$ is $T$-periodic defined as

$$
r(t)= \begin{cases}0, & t \in(0, \pi], \\ 1+\cos t, & t \in(-\pi, 0] .\end{cases}
$$

From (3.55) we see that $\varphi(t)=-1-\frac{1}{2} \sin t$. Obviously, $\varphi, r \in L([0, T], R)$, and condition (1) is satisfied. By direct calculation we have

$$
\frac{T}{4} \int_{0}^{T}[\varphi(s)]_{+}(s) d s=0<1 .
$$

This implies that condition (2) is satisfied. Furthermore, we can also get the equalities

$$
\int_{0}^{T}[\varphi(s)]_{-} d s=\int_{0}^{2 \pi}\left(1+\frac{1}{2} \sin t\right) d s=2 \pi
$$

and

$$
\int_{0}^{T}[\varphi(s)]_{+} d s=0
$$

which results in

$$
\begin{aligned}
& \frac{T}{4} \int_{0}^{T}[\varphi(s)]_{+} d s \int_{0}^{T}[\varphi(s)]_{-} d s=0, \\
& \int_{0}^{T}[\varphi(s)]_{-} d s-\int_{0}^{T}[\varphi(s)]_{+} d s=2 \pi .
\end{aligned}
$$

Consequently, all the conditions of Theorem 3.8 are satisfied, so that by this theorem there exists at least one positive $T$-periodic solution to equation (3.55).

Remark 3.1 Obviously, the conclusions associated with Examples 3.1-3.4 can be obtained neither by using the results of [7] nor by using the results of [16] (see Theorem 1.1), since the singularity term $\frac{r(t)}{x^{\mu}}$ is nonautonomous. Furthermore, even if $f(x) \equiv 0$ for $x \in(0,+\infty)$, the above conclusion associated with Example 3.4 cannot be deduced from the main theorem of [17] (Theorem 1 of [17]). This is due to the fact that the condition of $\delta \in[0,1$ ) is required in [17].

\section{Conclusions}

In this paper, we study the periodic problem for Liénard equations with a singularity of attractive type in the case of $r(t) \geq 0$ for a.e. $t \in[0, T]$. The proofs of main results are based on the method of upper and lower functions. It is interesting that the singularity term $\frac{r(t)}{x^{\mu}}$ in (1.7) is nonautonomous, which generalizes the corresponding results in the known literature where $r(t)$ is a constant function. In the next research, we will continue 
to study the periodic problem to the singular equation like (1.7) where $r(t)$ is a changingsign function.

\author{
Acknowledgements \\ The authors are grateful to anonymous referees for their constructive comments and suggestions, which have greatly \\ improved this paper.
}

\title{
Funding
}

The authors are gratefully acknowledge support from NSF of China (No. 11271197).

Availability of data and materials

Not applicable.

\section{Ethics approval and consent to participate}

Not applicable.

\section{Competing interests}

The authors declare that there is no conflict of interest regarding the publication of this manuscript. The authors declare that they have no competing interests.

\section{Consent for publication}

Not applicable.

\section{Authors' contributions}

Both authors contributed equally to this work. Both authors read and approved the final manuscript.

\section{Publisher's Note}

Springer Nature remains neutral with regard to jurisdictional claims in published maps and institutional affiliations.

Received: 8 February 2018 Accepted: 19 June 2018 Published online: 28 June 2018

\section{References}

1. Torres, P.J.: Mathematical Models with Singularities-A Zoo of Singular Creatures. Atlantis Press, Amsterdam (2015). https://doi.org/10.2991/978-94-6239-106-2

2. Bevc, V., Palmer, J.L., Süsskind, C.: On the design of the transition region of axi-symmetric magnetically focused beam valves. J. Br. Inst. Radio Eng. 18, 696-708 (1958)

3. Ye, Y., Wang, X.: Nonlinear differential equations in electron beam focusing theory. Acta Math. Appl. Sin. 1, 13-41 (1978) (in Chinese)

4. Huang, J., Ruan, S., Song, J.: Bifurcations in a predator-prey system of Leslie type with generalized Holling type III functional response. J. Differ. Equ. 257(6), 1721-1752 (2014)

5. Plesset, M.S., Prosperetti, A.: Bubble dynamic and cavitation. Annu. Rev. Fluid Mech. 9, 145-185 (1977)

6. Ghergu, M., Radulescu, V.: Singular Elliptic Problems: Bifurcation and Asymptotic Analysis. Oxford Lecture Series in Mathematics and Its Applications, vol. 37. The Clarendon Press, Oxford University Press, Oxford (2008)

7. Jebelean, P., Mawhin, J.: Periodic solutions of singular nonlinear differential perturbations of the ordinary $p$-Laplacian. Adv. Nonlinear Stud. 2(3), 299-312 (2002)

8. Torres, P.J.: Weak singularities may help periodic solutions to exist. J. Differ. Equ. 232, 277-284 (2007)

9. Li, X., Zhang, Z.: Periodic solutions for second order differential equations with a singular nonlinearity. Nonlinear Anal. 69, 3866-3876 (2008)

10. Lu, S., Wang, Y., Guo, Y.: Existence of periodic solutions of Liénard equation with a singularity of repulsive type. Bound Value Probl. 2017, Article ID 95 (2017). https://doi.org/10.1186/s13661-017-0826-5

11. Zhang, M.: Periodic solutions of Liénard equations with singular forces of repulsive type. J. Math. Anal. Appl. 203(1), 254-269 (1996). https://doi.org/10.1006/jmaa.1996.0378

12. Martins, R.: Existence of periodic solutions for second-order differential equations with singularities and the strong force condition. J. Math. Anal. Appl. 317, 1-13 (2006)

13. Lu, S.: A new result on the existence of periodic solutions for Liénard equations with a singularity of repulsive type. J. Inequal. Appl. 2017, Article ID 37 (2017). https://doi.org/10.1186/s13660-016-1285-8

14. Gutiérrez, A., Torres, P.J.: The Lazer-Solimini equation with state-dependent delay. Appl. Math. Lett. 25, 643-647 (2012)

15. Kong, F., Lu, S., Liang, Z.: Existence of periodic solutions for neutral Liénard differential equations with a singularity. Electron. J. Differ. Equ. 242, 1 (2015)

16. Hakl, R., Torres, P.J., Zamora, M.: Periodic solutions of singular second order differential equations: upper and lower functions. Nonlinear Anal. 74, 7078-7093 (2011)

17. Hakl, R., Zamora, M.: Existence and uniqueness of a periodic solution to an indefinite attractive singular equation. Ann. Mat. 195, 995-1009 (2016)

18. Lazer, A.C., Solimini, S.: On periodic solutions of nonlinear differential equations with singularities. Proc. Am. Math. Soc. 99, 109-114 (1987)

19. Hakl, R., Zamora, M.: On the open problems connected to the results of Lazer and Solimini. Proc. R. Soc. Edinb., Sect. A, Math. 144, 109-118 (2014) 
20. Hakl, R., Zamora, M.: Periodic solutions to the Liénard type equations with phase attractive singularities. Bound. Value Probl. 2013, Article ID 47 (2013). https://doi.org/10.1186/1687-2770-2013-47

21. Torres, P.J.: Weak singularities may help periodic solutions to exist. J. Differ. Equ. 232, 277-284 (2007)

22. Chu, J., Torres, P.J., Zhang, M.: Periodic solutions of second order non-autonomous singular dynamical systems. J. Differ. Equ. 239, 196-212 (2007)

23. Li, X., Zhang, Z.: Periodic solutions for second order differential equations with a singular nonlinearity. Nonlinear Anal. 69, 3866-3876 (2008)

24. Chu, J., Torres, P.J., Wang, F.: Twist periodic solutions for differential equations with a combined attractive-repulsive singularity. J. Math. Anal. Appl. 4371, 1070-1083 (2016). https://doi.org/10.1016/j.jmaa.2016.01.057

25. Hakl, R., Torres, P.J.: On periodic solutions of second order differential equations with attractive-repulsive singularities J. Differ. Equ. 248, 111-126 (2010)

26. Cheng, Z., Ren, J.: Multiplicity results of positive solutions for fourth-order nonlinear differential equation with singularity. Math. Methods Appl. Sci. 38, 5284-5304 (2016)

27. Cheng, Z., Ren, J.: Positive solutions for fourth-order singular nonlinear differential equation with variable-coefficient. Math. Methods Appl. Sci. 39, 2251-2274 (2016)

28. Chu, J., Liang, Z., Liao, F., Lu, S.: Existence and stability of periodic solutions for relativistic singular equations. Commun Pure Appl. Anal. 16(2), 591-609 (2017)

29. Lu, S., Guo, Y., Chen, L.: Periodic solutions for Liénard equation with singularities. J. Nonlinear Sci. Appl. 11, 403-415 (2018)

30. Abatangelo, N.: Very large solutions for the fractional Laplacian: towards a fractional Keller-Osserman condition. Adv. Nonlinear Anal. 6, 383-405 (2017)

31. Agarwal, R., Cetin, E.: Unbounded solutions of third order three-point boundary value problems on a half-line. Adv. Nonlinear Anal. 5, 105-119 (2016). https://doi.org/10.1515/anona-2015-0043

32. De Coster, C., Habets, P.: The lower and upper solutions method for boundary value problems. In: Handbook of Differential Equations, pp. 69-160. North-Holland, Amsterdam (2004)

33. Rachunková, I., Stanĕk, S., Tvrdý, M.: Solvability of Nonlinear Singular Problems for Ordinary Differential Equations. Contemporary Mathematics and Its Applications, vol. 5. Hindawi Publishing Corporation, New York (2008)

\section{Submit your manuscript to a SpringerOpen ${ }^{\circ}$ journal and benefit from:}

- Convenient online submission

- Rigorous peer review

- Open access: articles freely available online

- High visibility within the field

- Retaining the copyright to your article

Submit your next manuscript at $>$ springeropen.com 